\title{
Polymorphisms and expression of the WNT8A gene in Hirschsprung's disease
}

\author{
HONG GAO, DONG CHEN, XIAOMEI LIU, MEI WU, JIE MI and WEILIN WANG \\ Laboratory of Pediatric Congenital Malformation, Ministry of Public Health, \\ Shengjing Hospital of China Medical University, Shenyang, Liaoning 110004, P.R. China
}

Received March 23, 2013; Accepted July 1, 2013

DOI: $10.3892 /$ ijmm.2013.1433

\begin{abstract}
Hirschsprung's disease (HSCR) is a congenital disorder characterized by an absence of intrinsic ganglion cells in the nerves forming the plexus of the lower intestine. The WNT signaling pathway is considered to play an important role in embryonic development. In the present study, we analyzed 2 polymorphisms of the WNT8A gene (rs78301778 and rs6596422) to determine their association with the risk and development of HSCR. Allele frequencies and genotype distributions were analyzed by sequence analysis in patients with HSCR and normal controls. Using real-time PCR, western blot analysis and immunohistochemistry, we detected the mRNA and protein expression of WNT8A in patients with HSCR. The data indicated that the differences in genotype distributions and allele frequencies of rs78301778 and rs6596422 between various clinical classifications were statistically significant. The analysis of the mRNA and protein expression of WNT8A revealed that the expression of WNT8A was increased in the stenotic colon segments compared with the normal colon segments. In conclusion, the data presented in this study suggest that the WNT8A gene is involved in the susceptibility to HSCR, and plays an important role in the occurrence and development of HSCR. These findings warrant further investigation.
\end{abstract}

\section{Introduction}

Hirschsprung's disease (HSCR, aganglionic megacolon) represents the main genetic cause of functional intestinal obstruction with an incidence of 1 in 5,000 live births (1). HSCR is caused by multiple factors which affect the development of ganglion cells at different stages of development, and genetic factors have been demonstrated to play an important role in the development of HSCR (2). Previous studies have shown that

Correspondence to: Professor Hong Gao, Laboratory of Pediatric Congenital Malformation, Ministry of Public Health, Shengjing Hospital of China Medical University, 36 Sanhao Street, Heping District, Shenyang, Liaoning 110004, P.R. China

E-mail: gaohong515@sina.com

Key words: Hirschsprung's disease, WNT8A gene, polymorphism, expression the genetic etiology of neurocristopathy is complex and several genes may be involved in the development of HSCR (3-5). To date, 10 genes and 5 loci have been found to be involved in the development of HSCR. It is well known that ret proto-oncogene (RET) and endothelin receptor type B (EDNRB) are primary genes involved in the development of HSCR. RET mutations are associated with the development of HSCR in 50\% of cases in a familial series, but only $3 \%$ of sporadic HSCR cases carry RET mutations (5). Apart from RET and EDNRB, other genes have been identified in sporadic affected individuals, such as endothelin 3 (EDN3), endothelin converting enzyme 1 (ECE1), SRY (sex determining region Y)-box 10 (SOX10), glial cell-derived neurotrophic factor (GDNF), neurturin (NTN), paired-like homeobox 2b (PHOX2B), transcription factor 4 (TCF4) and Smad-interacting protein 1 (SIP1, also known as ZFHX1B) (6-12). Thus, HSCR has become a model of a complex polygenic disorder in which the interaction of different genes is still being elucidated.

The aim of this study was to determine whether genetic variations in the WNT8A gene are associated with HSCR and to examine the biological expression levels in Chinese patients with HSCR. Two single nucleotide polymorphisms (SNPs) in the WNT8A gene (rs78301778 and rs6596422) were selected and analyzed in a group of patients with HSCR and matched control samples. We further detected the differential expression of WNT8A by real-time PCR, western blot analysis and immunohistochemical staining.

\section{Patients and methods}

Patients. This study was approved by the Ethics Committee of China Medical University, Shenyang, China (no. 2013PS07K). Blood samples were collected from from $180 \mathrm{HSCR}$ patients at the Department of Pediatric Surgery, Shengjing Hospital of China Medical University. Patients with familial constipation and a history of other congenital GI tract malformations were excluded from this study. The age of the patients ranged from 0.5 to 3.5 years; our patient cohort included 141 males and 39 females (average age, $1.5 \pm 0.3$ years); these patients were recruited as the HSCR group. An additional 180 healthy children that matched the HSCR group in age and gender were used as the control group (average age, $2 \pm 0.5$ years). The control group had no history of constipation. Tissue samples (the stenotic and normal colon segment) were obtained from 60 HSCR patients. 
Genomic DNA extraction. Venous blood (200 $\mu \mathrm{l})$ was obtained from the study participants using EDTA as an anticoagulant. Genomic DNA of peripheral blood white blood cells (WBCs) was extracted according to the QIAamp ${ }^{\circledR}$ DNA Blood Mini Kit Handbook. For the present study, the absorbance value at $260 / 280 \mathrm{~nm}\left(\mathrm{~A}_{260} / \mathrm{A}_{280}\right)$ ranged from 1.6 to 2.0 , which met the requirements for further experiments.

Detection of WNT8A genotype. Genomic DNA from peripheral blood was obtained with QIAamp Blood kits (Takara, Dalian, China) using standard methods (13). Genotypes were analyzed using PCR and direct sequencing, as described below, performed without knowledge of the case-control status of the patients. The PCR primers used were as follows: rs78301778-1, GCC TCT GCT TTG GGT AAT; rs78301778-2, GTG TCC CTC AGC CTT TCT (product size, 278 bp); rs6596422-1, TCC CTA CTC AGA GCC ATT C; rs6596422-2, TGA CCG TAC AGC ACC ACT (product size, 499 bp).

Real-time PCR. Total RNA was extracted from the stenotic and normal colon segment tissues from patients with HSCR using TRIzol reagent (Life Technologies Corp., Carlsbad, CA, USA) according to the manufacturer's instructions. The primers used for PCR were as follows: WNT8A-v1, AGA GGC GGA ACT GA; and WNT8A-v2, TCC CAC CTG GAT GT. $\beta$-actin (DR3783; Takara) was used as the loading control to demonstrate the equivalent amounts of cDNA in each lane in real-time PCR. The relative mRNA levels for each sample were calculated using the $2^{-\Delta \Delta C t}$ method.

Western blot analysis. Equal amounts of total protein from the tissues were separated on SDS-polyacrylamide gels and then electrotransferred onto PVDF membranes (Millipore, Billerica, MA, USA). The blots were incubated with rabbit polyclonal WNT8A antibody (1:200; Novus Biologicals, Littleton, CO, USA; Catalog number: 23050002, $40 \mathrm{kDa}$ ) overnight at $4^{\circ} \mathrm{C}$; washed, incubated with horseradish peroxidase-linked secondary antibodies $(1: 2,000)$ for $1 \mathrm{~h}$ at room temperature and detected using an enhanced chemiluminescence (ECL) kit. Detected bands were quantified using Gel-pro 4.0 software (Media Cybernetics, L.P. Gel-Pro Analyzer; Media Cybernetics, Inc. Rockville, MD, USA).

Immunohistochemical staining. Consecutive paraffin wax-embedded tissue sections (4-7 $\mu \mathrm{m})$ were dewaxed and rehydrated. Antigen retrieval was performed by pre-treatment of the slides in citrate buffer ( $\mathrm{pH}$ 6.0) in a microwave oven for $12 \mathrm{~h}$. Thereafter, the slides were cooled to room temperature in deionised water for $5 \mathrm{~h}$. Endogenous peroxidase activity was quenched by incubating the slides in methanol containing $0.6 \%$ hydrogen peroxide, followed by washing in deionised water for $4 \mathrm{~h}$, after which the sections were incubated for $1 \mathrm{~h}$ at room temperature with normal goat serum. The slides were then set on a flat surface, and the sections were coated in a solution of rabbit polyclonal WNT8A antibody (1:200; Novus Biologicals) at room temperature for $30 \mathrm{~min}$. The slides then were coated in a solution of goat anti-rabbit antibody $(1: 2,000$; Dako, Glostrup, Denmark) for $30 \mathrm{~min}$, and finally in a solution of streptavidin-horseradish peroxidase (LSAB2 System; Dako) for $30 \mathrm{~min}$. Antibody detection was visualized using

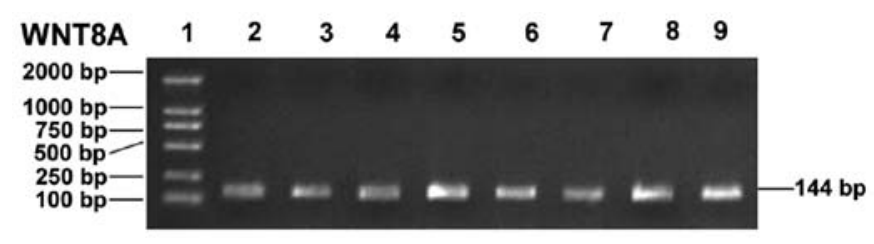

Figure 1. Agarose gel electrophoresis of PCR products of the WNT8A gene. Lane 1, Marker DL 2000; lanes 2-9, PCR amplification products of the WNT8A gene.

a substrate-chromogen solution (LSAB2 System; Dako) for 5 to 30 min that was counterstained with Mayer's hematoxylin (Merck, Darmstadt, Germany) for $1 \mathrm{~min}$. The density of the positively stained area was calculated at $\mathrm{x} 400$ magnification as the sum of the areas occupied by the positively stained area of the plexus.

Statistical analysis. The $\chi^{2}$ test was performed to determine whether each polymorphism was in the Hardy-Weinberg equilibrium within the control and patient groups. The relative density of the bands was expressed as the $2^{-\Delta \Delta \mathrm{Ct}}$ value of each sample as parametric data for quantitative real-time PCR, western blot analysis and immunohistochemistry. Statistical significance was determined using the Student's t-test; a P-value $<0.05$ was considered to indicate a statistically significant difference.

\section{Results}

PCR amplification of WNT8A gene. PCR amplification was successfully performed. The amplified segment of the WNT8A gene was $144 \mathrm{bp}$, which was in accordance with theoretical lengths. The amount of amplified products was large and no non-specific bands appeared (Fig. 1).

Distribution of WNT8A allele and genotype frequencies in patients with HSCR and controls. Genotype distributions in the 2 SNPs were in accordance with the Hardy-Weinberg equilibrium (Fig. 2). As illustrated in Table I, the WNT8A rs78301778 null genotype was associated with a greater risk of HSCR (Table I). The WNT8A rs6596422 null genotype was also associated with a greater risk of HSCR (Table I). The allele frequencies at SNP rs78301778 revealed a significant association of allele T with HSCR (Table I). At the genotype level, HSCR was negatively associated with TT homozygosity and positively associated with GT heterozygosity and GG homozygosity $(\mathrm{P}=0.001)$, which indicated that the risk of HSCR was significantly higher among patients with the GT or GG genotype. The allele frequencies at SNP rs6596422 revealed a significant association of allele $\mathrm{G}$ with HSCR $(\mathrm{P}=0.001)$. At the genotype level, HSCR was negatively associated with GG homozygosity and positively associated with AG heterozygosity and AA homozygosity $(\mathrm{P}=0.004)$, which indicated that the risk of HSCR was significantly higher among patients with the AG or AA genotype. The differences in genotype and allele distributions of rs 78301778 and rs6596422 between various clinical classifications were statistically significant (Table II). 
A: rs78301778

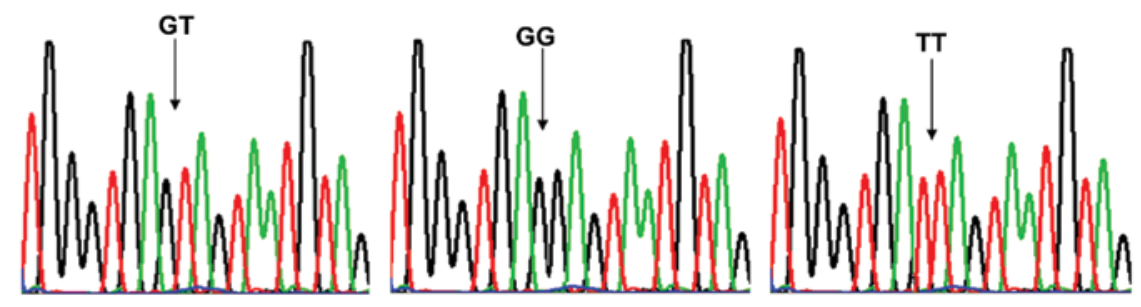

B: rs6596422

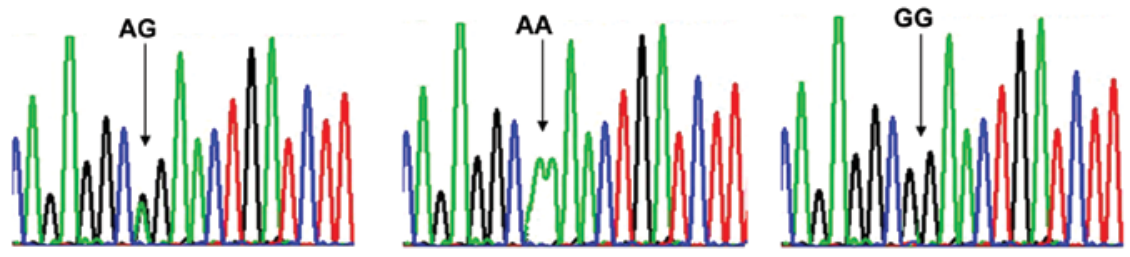

Figure 2. Results from sequencing analysis of different genotypes of the rs78301778 and rs6596422 polymorphisms. (A) GT, GG and TT were the genotypes detected for rs78301778. (B) AG, AA and GG were the genotypes detected for rs6596422. The arrow denotes the single nucleotide polymorphism location.

Table I. Allele frequency and genotype distribution in patients with HSCR and controls.

\begin{tabular}{lcccccc}
\hline Polymorphism & Type & HSCR & Controls & $\chi^{2}$ & P-value & OR (95\% CI) \\
\hline rs78301778 & GT & 87 & 91 & - & - & - \\
& GG & 69 & 63 & 12.302 & 0.001 & $2.233(1.421-3.509)$ \\
& TT & 24 & 26 & 0.858 & 0.353 & $0.724(0.366-1.435$ \\
& G & 225 & 217 & - & - & - \\
rs6596422 & T & 135 & 143 & 16.569 & 0.001 & $0.525(0.384-0.717)$ \\
& AG & 65 & 82 & - & - & - \\
& AA & 98 & 64 & 8.191 & 0.004 & $0.518(0.329-0.814)$ \\
& GG & 17 & 34 & 1.849 & 0.174 & $1.585(0.814-3.089)$ \\
& A & 261 & 210 & - & - & - \\
& G & 99 & 150 & 15.968 & 0.001 & $1.883(1.378-2.573)$
\end{tabular}

HSCR, Hirschsprung's disease; OR, odds ratio; CI, confidence interval.

Table II. Allele frequency and genotype distribution in patients with HSCR and controls.

\begin{tabular}{|c|c|c|c|c|c|c|c|}
\hline \multirow[b]{2}{*}{ Polymorphism } & \multirow[b]{2}{*}{ Group } & \multirow[b]{2}{*}{ Case (n) } & \multicolumn{3}{|c|}{ Genotype distribution (\%) } & \multicolumn{2}{|c|}{ Allele frequency $(\%)$} \\
\hline & & & GT & GG & TT & G & $\mathrm{T}$ \\
\hline \multirow[t]{3}{*}{ rs78301778 } & HSCR & 180 & $91(50.55)$ & $59(32.78)$ & $30(16.67)$ & $209(58.06)$ & $151(41.94)$ \\
\hline & Controls & 180 & $67(37.22)$ & $97(53.89)$ & $16(8.89)$ & $261(72.50)$ & $99(27.50)$ \\
\hline & & & \multicolumn{2}{|c|}{$\chi^{2}=17.163$} & 001 & $\chi^{2}=16.569$ & $\mathrm{P}=0.001$ \\
\hline
\end{tabular}

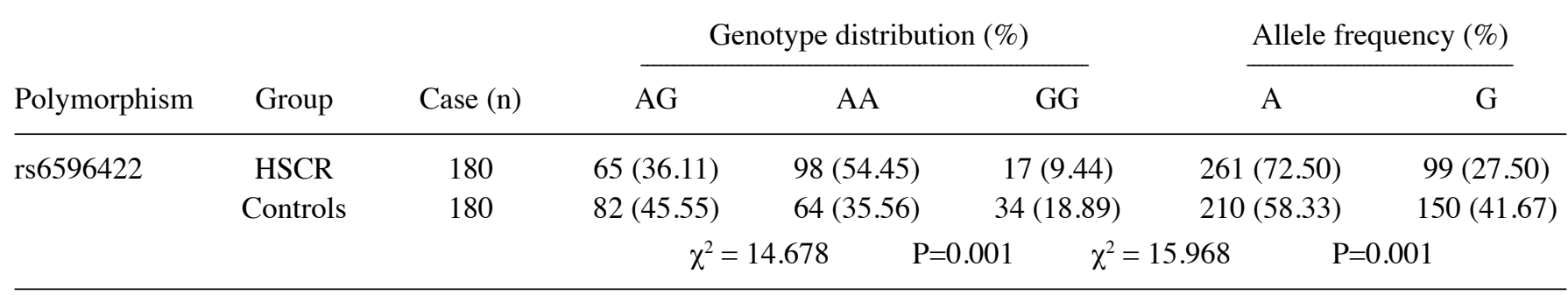

HSCR, Hirschsprung's disease. 


\section{A: rs78301778}
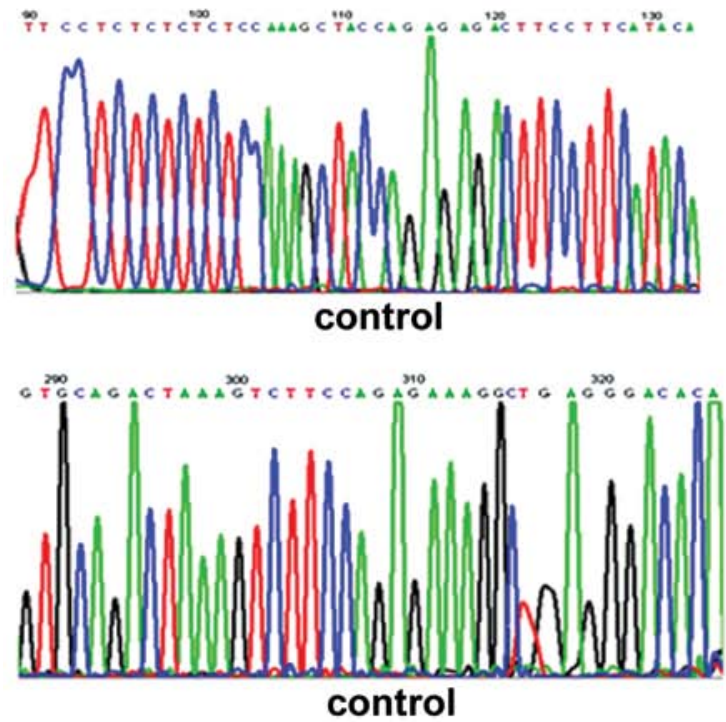

\section{B: rs6596422}
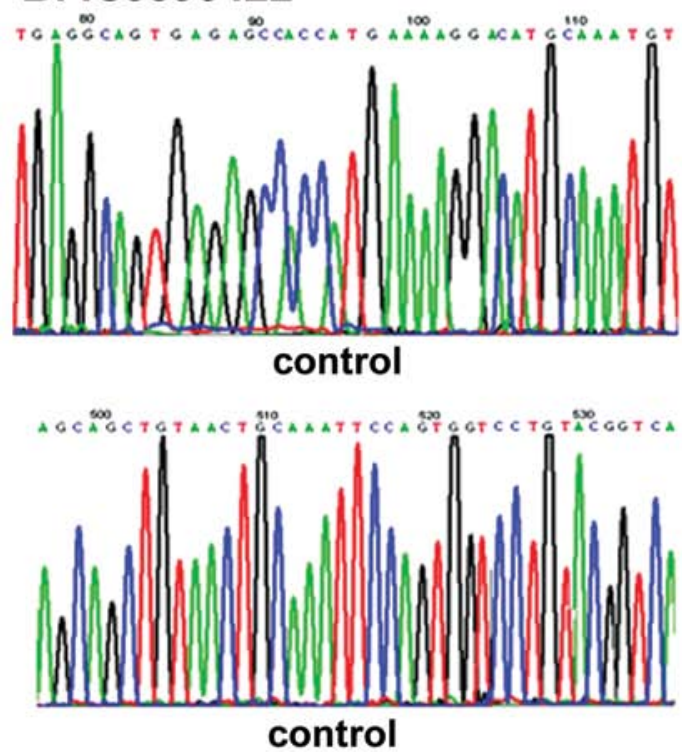

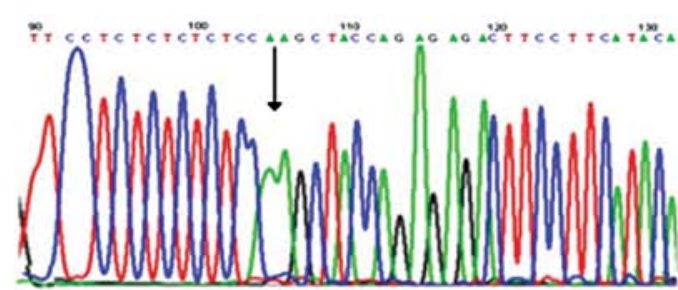

patient $A<105$
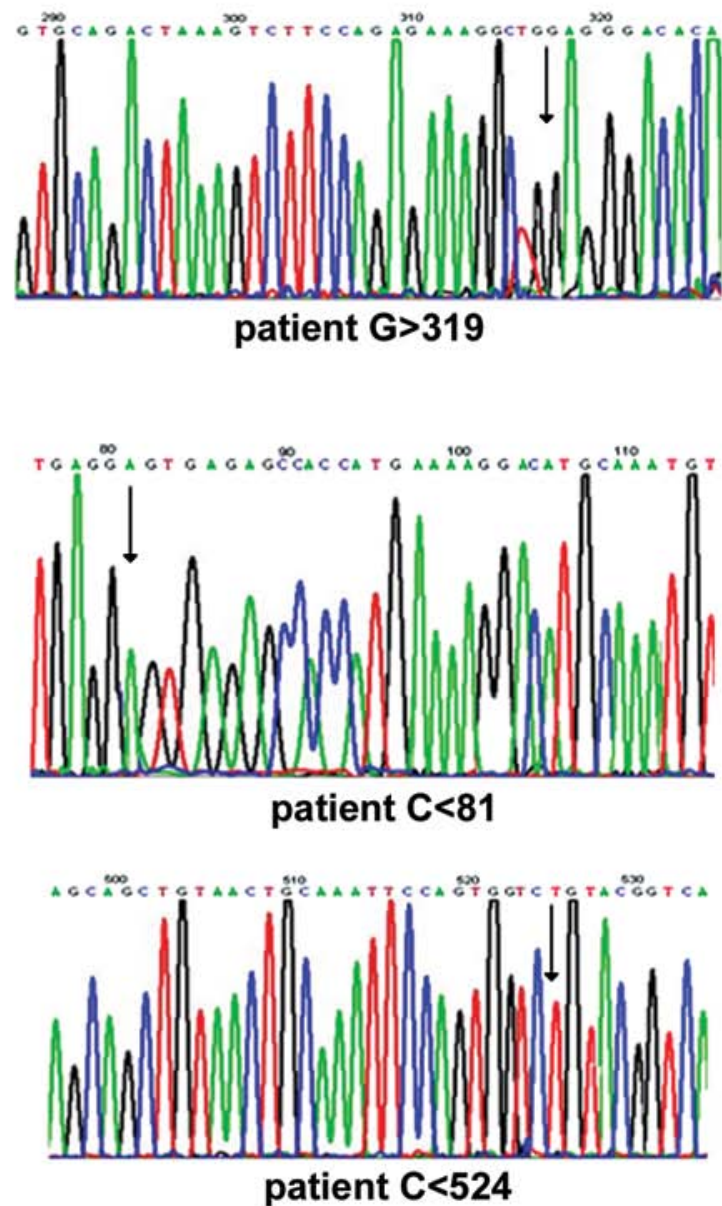

Figure 3. A novel mutation in the WNT8A gene. (A) Sequencing analysis of rs78301778 in the patient and the control group. Arrows denote the codon point. (B) Sequencing analysis of rs6596422 in the patient and the control group. Arrows denote the codon point.

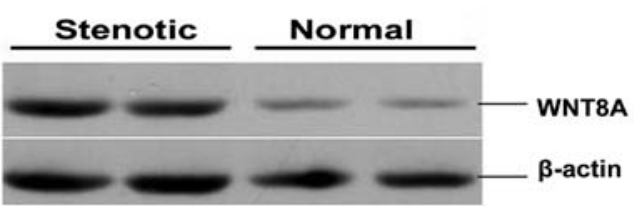

Figure 4. Western blot analysis of WNT8A expression in patients with Hirschsprung's disease (HSCR). The relative protein content of WNT8A was higher in the stenotic colon segments than in the normal colon segments.

Sequence analysis revealed that the TT genotype in the rs78301778 polymorphism lacks one 'A' at codon 105 , the GT genotype in rs78301778 lacks one ' $\mathrm{C}$ ' at codon 317 , and the GG genotype of the rs78301778 polymorphism has an extra 'G' at codon 309. Sequence analysis also demonstrated that the
AG genotype in the rs 6596422 polymorphism lacks one ' $\mathrm{C}$ ' at codon 81 and the GG genotype in rs6596422 also lacks one 'C' at codon 524 (Fig. 3).

Real-time PCR. To detect any changes at the transcriptional level of the WNT8A gene, we compared the mRNA levels by performing real-time PCR. The mRNA level of WNT8A was 3 -fold higher in the stenotic colon segments than in the normal colon segments $(n=60, P<0.001)$.

Protein analysis. We selected the WNT8A protein based on its biological functions and confirmed alteration in its expression in colon tissue from patients with HSCR by western blot analysis. The protein level of WNT8A was higher in the stenotic colon segments than in the normal colon segments (Fig. 4). 

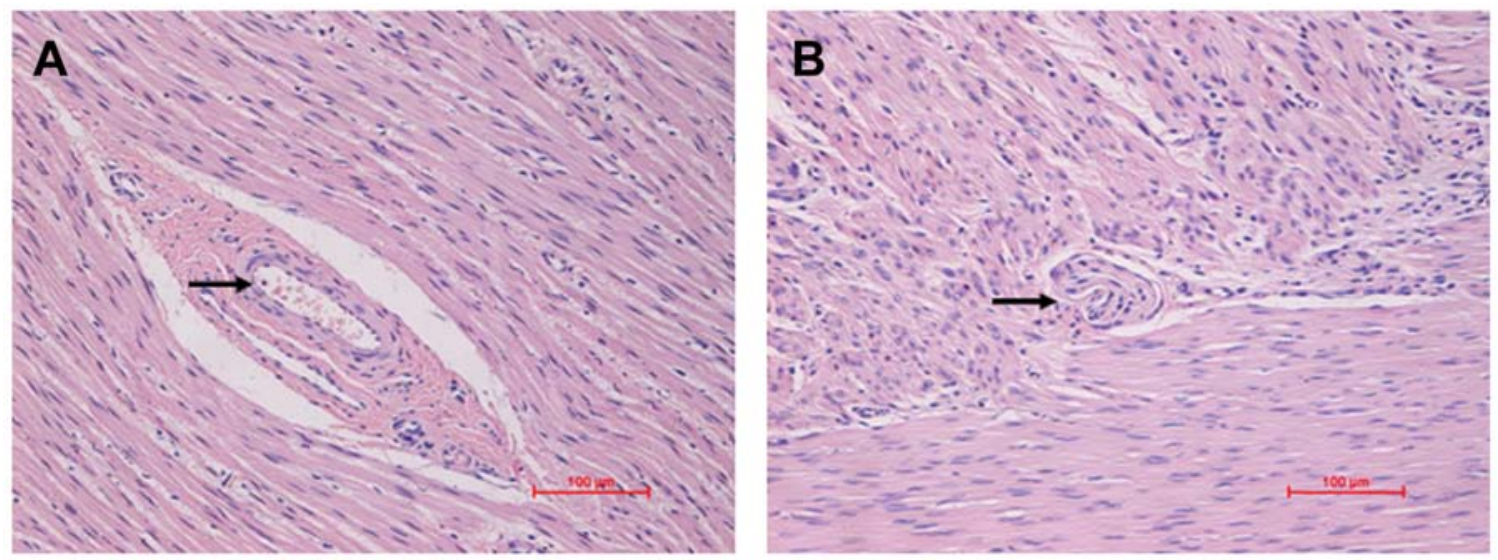

Figure 5. H\&E staining of samples obtained from patients with Hirschsprung's disease (HSCR). (A) Ganglionic segment from a patient with HSCR. The arrow shows the ganglionic cells (B) Aganglionic segment from a patient with HSCR. The arrow shows fibrous tissue hyperplasia where the ganglionic cell disappears. $\mathrm{H} \& \mathrm{E}$ staining (original magnification, $\mathrm{x} 200$ ).
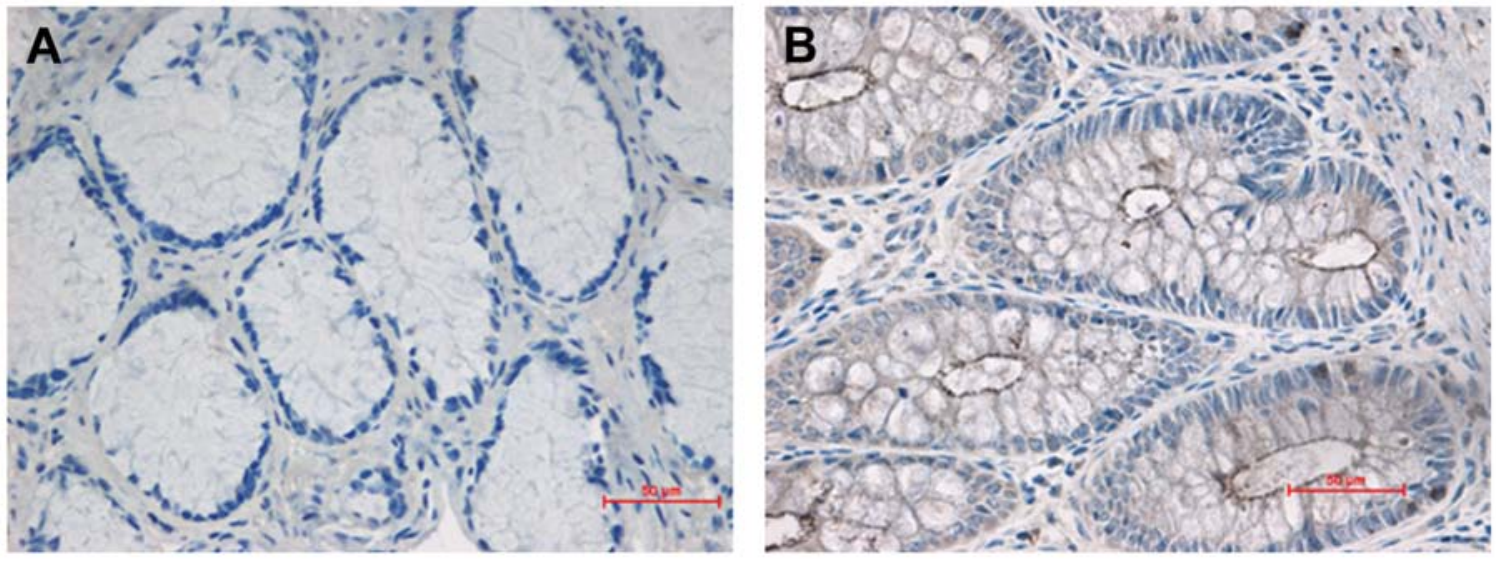

Figure 6. Distribution and expression of WNT8A detected by immunohistochemistry in patients with Hirschsprung's disease (HSCR) (original magnification, $\mathrm{x} 400$ ). (A) WNT8A staining in the mucosal layer and muscular layer of the normal colon segment tissues. (B) WNT8A staining in the mucosal layer and muscular layer of the stenotic colon segment tissues (original magnification, $\mathrm{x} 400$ ).

The protein expression level of WNT8A was $269.19 \pm 20.41$ and 147.19 \pm 15.27 in stenotic and normal colon segment tissues $(\mathrm{n}=60, \mathrm{P}<0.01)$, respectively.

Immunohistochemistry. Histopathological examination revealed that in the colon tissue from patients with HSCR, there was a loss of focal ganglion cells in the colon tissue, which is a common characterization in HSCR. The stenotic colon segment was defined by the loss of focal ganglion cells in the colon, as shown by H\&E staining (Fig. 5). To further investigate the distribution and expression of the WNT8A protein in colon tissue, we performed immunohistochemical staining. WNT8A was located in the mucosal layer and muscular layer of the colon segment tissues. The staining of WNT8A was much stronger (brown) in the stenotic colon segment tissues than in the normal colon segment tissues (colorless or light yellow) (Fig. 6).

\section{Discussion}

The WNT signaling pathway plays an important role in embryonic development. A critical factor to mesoderm development is the secreted ligand, WNT8A. At the onset of gastrulation, WNT8A signaling prevents the dorsal organizer from expansion by regulating the expression of the transcriptional repressors, vent, vox, and ved, in the ventrolateral mesoderm (14). During and after gastrulation, WNT8A functions downstream of brachyury-related T-box transcription factors, regulating posterior mesoderm maintenance and proliferation $(15,16)$. WNT8A signaling is also crucial to the nervous system in anteroposterior patterning $(17,18)$. Thus, WNT8A expression is a critical component of the mesoderm gene that regulates the signaling network with ramifications for global embryonic axis patterning. Consequently, understanding the transcriptional regulation of WNT8A is a critical step in unraveling multiple aspects of early vertebrate development.

As HSCR is a multifactorial congenital disorder, the cumulative genetic effects that result in an individual phenotypic variation play a crucial role in its development. Therefore, it is important to assess whether WNT8A polymorphisms are associated with HSCR susceptibility. The aim of the present study was to examine polymorphic markers of the WNT8A gene to determine their association with the risk and development of HSCR in Chinese individuals. DNA was extracted from whole blood samples, and WNT8A polymorphisms were analyzed by PCR. Associations between specific genotypes and the development of HSCR were examined by logistic regression 
analysis to calculate the odds ratio (OR) and 95\% confidence intervals (CI). The risk of HSCR increased as the number of putative high-risk genotypes increased for the combined genotypes of WNT8A heterozygosity. In conclusion, the results obtained in this study clearly suggest that the susceptible factor related to different WNT8A polymorphisms is predisposing risk factor for HSCR. We observed that the WNT8A gene polymorphisms (rs78301778 and rs6596422) are associated with an increased risk of HSCR in our study sample. The differences in genotypes and allele distributions of rs78301778 and rs6596422 between various clinical classifications were statistically significant. Moreover, sequence analysis revealed that the WNT8A gene may influence the risk of this common developmental anomaly.

In addition, we confirmed WNT8A expression by realtime PCR, western blot analysis and immunohistochemical staining. The mRNA and protein expression level of WNT8A was significantly higher in the stenotic colon segments than in the normal colon segments. The immunohistochemical staining of WNT8A was much stronger (brown) in the stenotic colon segment tissues than in the normal colon segment tissues (colorless or light yellow).

In conclusion, our study demonstrates that polymorphic variants of WNT8A may be involved in the development of HSCR. We also detected WNT8A as a differentially expressed gene in the stenotic and normal colon segments obtained from patients with HSCR. Our study may provide new insight into the development of HSCR.

\section{Acknowledgements}

This study was supported by grants from the National Natural Science Foundation of China (grant no. 30772277). We thank Professor Zhijie Li of the Central Laboratory, Shengjing Hospital Affiliated to China Medical University for reviewing the manuscript.

\section{References}

1. Amiel J, Sproat-Emison E, Garcia-Barcelo M, Lantieri F, Burzynski G, Borrego S, Pelet A, Arnold S, Miao X, Griseri P, Brooks AS, Antinolo G, de Pontual L, Clement-Ziza M, Munnich A, Kashuk C, West K, Wong KK, Lyonnet S, Chakravarti A, Tam PK, Ceccherini I, Hofstra RM and Fernandez R: Hirschsprung disease, associated syndromes and genetics: a review. J Med Genet 45: 1-14, 2008.
2. Grundy D and Schemann M: Enteric nervous system. Curr Opin Gastroenterol 21: 176-182, 2005.

3. Lantieri F, Griseri P and Ceccherini I: Molecular mechanisms of RET-induced Hirschsprung pathogenesis. Ann Med 38: 11-19, 2006.

4. Arighi E, Borrello MG and Sariola H: RET tyrosine kinase signaling in development and cancer. Cytokine Growth Factor Rev 16: 441-467, 2005.

5. Tam PK and Garcia-Barcelo M: Molecular genetics of Hirschsprung's disease. Semin Pediatr Surg 13: 236-248, 2004.

6. Sánchez-Mejías A, Fernández RM, López-Alonso M, Antiñolo G and Borrego S: New roles of EDNRB and EDN3 in the pathogenesis of Hirschsprung disease. Genet Med 12: 39-43, 2010.

7. Núñez-Torres R, Fernández RM, López-Alonso M, Antiñolo G and Borrego S: A novel study of copy number variations in Hirschsprung disease using the multiple ligation-dependent probe amplification (MLPA) technique. BMC Med Genet 10: $119,2009$.

8. Pan ZW, Lou J, Luo C, Yu L and Li JC: Association analysis of the SOX10 polymorphism with Hirschsprung disease in the Han Chinese population. J Pediatr Surg 46: 1930-1934, 2011.

9. Fernandez RM, Ruiz-Ferrer M, Lopez-Alonso M, Antiñolo G and Borrego S: Polymorphisms in the genes encoding the 4 RET ligands, GDNF, NTN, ARTN, PSPN, and susceptibility to Hirschsprung disease. J Pediatr Surg 43: 2042-2047, 2008.

10. Kwon MJ, Lee GH, Lee MK, Kim JY, Yoo HS, Ki CS, Chang YS, Kim JW and Park WS: PHOX2B mutations in patients with Ondine-Hirschsprung disease and a review of the literature. Eur J Pediatr 170: 1267-1271, 2011.

11. Jiang Q, Ho YY, Hao L, Nichols Berrios C and Chakravarti A: Copy number variants in candidate genes are genetic modifiers of Hirschsprung disease. PLoS One 6: e21219, 2011.

12. Gregory-Evans CY, Vieira H, Dalton R, Adams GG, Salt A, Gregory-Evans K: Ocular coloboma and high myopia with Hirschsprung disease associated with a novel ZFHX1B missense mutation and trisomy 21. Am J Med Genet A 131: 86-90, 2004.

13. Bai Y, Wang Z, Dai W, Li Q, Chen G, Cong N, Guan M and Li H: A six-generation Chinese family in haplogroup B4C1C exhibits high penetrance of $1555 \mathrm{~A}>\mathrm{G}$-induced hearing loss. BMC Med Genet 11: 129, 2010.

14. Ramel MC and Lekven AC: Repression of the vertebrate organizer by Wnt 8 is mediated by Vent and Vox. Development 131: 3991-4000, 2004.

15. Martin BL and Kimelman D: Regulation of canonical Wnt signaling by Brachyury is essential for posterior mesoderm formation. Dev Cell 15: 121-133, 2008.

16. Baker KD, Ramel MC and Lekven AC: A direct role for Wnt8 in ventrolateral mesoderm patterning. Dev Dyn 239: 2828-2836, 2010.

17. Erter CE, Wilm TP, Basler N, Wright CV and Solnica-Krezel L: Wnt 8 is required in lateral mesendodermal precursors for neural posteriorization in vivo. Development 128: 3571-3583, 2001.

18. Rhinn M, Lun K, Luz M, Werner M and Brand M: Positioning of the midbrain-hindbrain boundary organizer through global posteriorization of the neuroectoderm mediated by Wnt 8 signaling. Development 132: 1261-1272, 2005. 